\title{
Seismic Radiation from M-class Solar Flares
}

\author{
Diana Besliu-Ionescu ${ }^{1,2}$, Alina-C. Donea ${ }^{2}$, \\ Paul Cally ${ }^{2}$ and Charles Lindsey ${ }^{3}$
}

\author{
${ }^{1}$ Department of Solar Physics, Astronomical Institute of the Romanian Academy \\ Str. Cutitul de Argint Nr.5, RO-040557, Bucharest, Romania \\ email: diana.besliu@gmail.com \\ ${ }^{2}$ C.S.P.A., School of Mathematical Sciences, Monash University \\ Victoria, AUSTRALIA 3800 \\ ${ }^{3}$ Colorado Research Associates Division, NorthWest Research Associates, Inc. \\ 3380 Mitchell Lane, Boulder, CO 80301, USA
}

\begin{abstract}
Helioseismic holography is a technique used to image the sources of seismic disturbances observed at the solar surface. It has been used to detect acoustic emission, known as sun quakes, radiated from X-class solar flares. Since the seismic power emitted by the X-class flares has proved to be independent of the strength of the flare, we have undertaking a systematic search for seismic signatures from M-class solar flares, observed by SOHO-MDI.

We have detected significant acoustic emission from a few M-class solar flares. Preliminary results of the survey of M-type solar flares studied so far is available at: aira.astro.ro/ deanna/ M.html.
\end{abstract}

Keywords. Flares, sun quakes, helioseismic holography

\section{Introduction}

The first sun quake occurred on 9 July 1996 and was discovered by Kosovichev \& Zharkova (1998). Donea, Braun \& Lindsey (1999) applied computational seismic holography to helioseismic observations of the flare to image its seismic source (the focus of the sun quake). A similar analysis applied to the two powerful flares of 28 and 29 October 2003 also showed pronounced seismic emission Donea \& Lindsey (2005). The acoustic signatures from these flares were somewhat less energetic than that of the X2.6 flare of 9 July 1996. They were, nevertheless, quite conspicuous and both of these flares were observed by a considerable array of other space-borne and ground-based facilities. These results suggest that the acoustic emission is driven by explosive heating and evaporation of the chromosphere, by high-energy particles travelling downward along the coronal magnetic field lines.

We have performed a systematic survey of the SOHO/MDI database for acoustic emission from solar flares, applying the technique of helioseismic holography Lindsey \& Braun (1997), Lindsey \& Braun (2000) to the observations. We analysed the X-class flares that occurred during the period of 1996-2005. Preliminary results of our survey are presented in Donea et al. (2005) and Besliu-Ionescu et al. (2005). Donea \& Lindsey (2005) suggested that significant seismic activity might possibly be excited by a relatively small flare (either a C- or a M-class flare) provided the energy is released suddenly into a relatively localized footpoint. We report the detection of seismic waves emitted from M-type flares. 


\section{Seismic Sources}

On 9 September 2001 at 20:40 UT, a M9.5 flare occurred at the coordinates S31E26 in active region AR 9608. The active region was quite bright in X-rays. The main spot had an extensive penumbra and a negative polarity, it was surrounded by positive polarity plages. The X-ray flux from the flare measured by GOES reached a peak at 20:46 UT.

Significant acoustic emission at $6 \mathrm{mHz}$ is visible in the penumbra of the main sunspot. A detectable Doppler signature is seen in the southern part of the penumbra of the main sunspot at the location of the $6 \mathrm{mHz}$ seismic source. The egression signature spatially coincides with the local disturbance in the source region that begins with a rapid downward depression of the photosphere. Remarkable is that the white-light observations of the flare show a kernel-like structure with a perfect spatial correspondence between the GONG intensity and the egression power kernel. Details about this seismic source can be read in Donea et al. (2006).

On 15 August 2004 at 12:34 UT, a M9.4 flare occurred at the coordinates S13W49 in active region AR 10656. The X-ray flux from the flare measured by GOES reached a peak at 12:41 UT. Significant acoustic emission at $6 \mathrm{mHz}$ is visible in the central sunspots group of the AR. A detectable Doppler signature of the active region at the location of the $6 \mathrm{mHz}$ seismic source. The egression signature spatially coincides with the neutral line of the central sunspots group.

\section{Conclusions}

- Moderately energetic flares can be more acoustically active than some of high energy.

- There is a strong association of compact seismic signatures from the M9.5 flare with sudden white-light flare emission.

- The conspicuous spatial and morphological correspondence between the white light and the seismic emission signatures suggests that the seismicity of the active region is related to deposition of energy into the photosphere, for which considerations of momentum balance are even more favorable than for waves of chromospheric origin.

- The persistence of a sudden, co-spatial white-light signature in flares where no protons are evident appears to be consistent with acoustic emission driven by photospheric back-warming of the low photosphere by radiation from a heated overlying chromosphere. Indeed, the sharp coincidence between acoustic emission and the sudden appearance of continuum emission offers strong support for the back-warming theory where it applies to the low photosphere.

- The fraction of energy emitted into the subphotosphere as seismic waves remains a small fraction of the total energy released in the flare. However, according to rough estimates based on simple models this appears to be consistent with seismic emission driven by photospheric heating.

\section{References}

Besliu-Ionescu, D., Donea, A., Cally, P. \& Lindsey, C. 2005, Proceedings of SPM-11, ESA SP CD-ROM.

Donea, A.-C. \& Lindsey, C. 2005, ApJ 630, 1168.

Donea, A.-C., Braun, D.C., \& Lindsey, C. 1999, ApJ 513, L143.

Donea, A., Besliu, D., Cally, P.,\& Lindsey, C. 2005, in J. Leibacher, H. Uitenbroek and B. Stein (ed.), Solar MHD: Theory and Observations - a High Spatial Resolution Perspective, ASP Conference Proceedings Series, in press.

Donea, A., Besliu-Ionescu, D., Cally, P., Lindsey, C. \& Zharkova, V. 2006, Solar Phys, accepted.

Kosovichev, A.G. \& Zharkova, V.V. 1998, Nature, 317.

Lindsey, C. \& Braun, D.C. 1997, Astrophys. J. 485, 895.

Lindsey, C. \& Braun, D.C. 2000, Solar Phys. 192, 261. 